\title{
Floquet scattering in parametric electron pumps
}

\author{
Sang Wook Kim \\ Max-Planck-Institut für Physik komplexer Systeme, Nöthnitzer Str. 38, D-01187 Dresden, Germany
}

(November 8, 2018)

\begin{abstract}
A Floquet scattering approach to parametric electron pumps is presented and compared with Brouwer's adiabatic scattering approach [Phys. Rev. B 58, R10135 (1998)] for a simple scattering model with two harmonically oscillating $\delta$-function barriers. For small strength of oscillating potentials these two approaches give exactly equivalent results while for large strength, these clearly deviate from each other. The validity of the adiabatic theory is also discussed by using the Wigner delay time obtained from the Floquet scattering matrix.

PACS number(s): 73.23.-b, 72.10.Bg, 73.50.Pz
\end{abstract}

\section{INTRODUCTION}

A parametric electron pump is a device that generates a dc current at zero bias potential through cyclic change of system parameters. The most direct way to create a dc current was originally proposed by Thouless [1], who considered a system subjected to a traveling wave potential. This can be realized for example with the help of surface acoustic waves [2]. Another possibility is to utilize quantum dots. In closed systems operating in the Coulomb blockade regime, integer number of electric charge can be transferred by sequential changes of barriers like a turnstile [3], whereas in open systems the electron pumping can be driven by adiabatic shape change in the confining potential or other parameters which affect the interference pattern of the coherent electrons in the device. After a cycle of the adiabatic shape change we return to the initial configuration, but the wavefunction may have its phase changed from the initial wavefunction. This is the so-called geometric or Berry's phase [1]. This additional phase is equivalent to some charges that pass through the quantum dot, namely, pumped charge [5,6]. Recently, adiabatic charge pumping in open quantum dots has attracted considerable attention [5] 12].

Switkes et al. report an experimental investigation of electron pumping through an open quantum dot under the shape deformation controlled by two ac gate voltages [8]. For weak pumping the dc voltage induced by the pumped dc current not only have a sinusoidal dependence on phase difference $\phi$ of the two ac voltages applied to the gates, but is also proportional to the square of the pumping strength $\lambda$. Many aspects of the experimental results can be understood in terms of Brouwer's scattering approach [7]. The change in the charge of the dot in response to a small variation of external parameter $\delta X$ is given by $\delta Q=e \sum \delta X_{i} d n / X_{i}$, where $d n / d X$ is the emissivity [13]. The pumped charge during each cycle can be determined by integrating $\delta Q$ along the closed path in the parameter space $\left\{X_{i}\right\}$ defined by one pumping cycle. Then, the pumped charge $Q$ is rewritten as an integral over the surface enclosed by the path by using Green's theorem, e.g. in two parameter case $Q=e \int_{A} d X_{1} d X_{2} \Pi\left(X_{1}, X_{2}\right)$. Assuming $\Pi$ is constant over small area of $\left(X_{1}, X_{2}\right)$, for weak pumping one can show the pumping current $I_{p} \propto \omega \lambda^{2} \sin \phi$, which exactly corresponds to what was observed in the experiment. For strong pumping, however, the dc voltage generated by the pumped current deviates from the sinusoidal dependence on $\phi$, and also departs from the $\lambda^{2}$ dependence. The non-sinusoidal dependence can be understood by taking into account that $\Pi$ is no longer constant over the integration area when $\lambda$ is considerably large. In Ref. [8] the anomalous $\lambda$ dependence was ascribed to the occurrence of significant heating and dephasing as a result of strong pumping.

The parametric electron pump is a time dependent system driven by (at least) two different time periodic perturbations with the same angular frequency and phase difference $\phi$. One can deal with this problem using not only adiabatic approximation exploited by Brouwer but also Floquet approach [14]. An oscillating potential can transfer an incoming electron of energy $E$ to Floquet side bands at $E \pm n \hbar \omega$, where $n$ is an integer and $\omega$ is the angular frequency of the oscillation. A scattering matrix for a time dependent system can be constructed from the interplay of these sidebands [15,16]. So far there have been relatively few works on non-adiabatic parametric electron pumps [17, 18], and the comparison between the adiabatic and the Floquet approach is still missing. We show, using a simple scattering model with two harmonically oscillating $\delta$-function barriers, that for small $\lambda$ these two approaches give exactly equivalent results for the pumped charge while for large $\lambda$ they are different. Even though the pumped current as a function of $\lambda$ obtained from these two approaches shows qualitatively similar behavior, the physical interpretation is completely different. This discrepancy in large $\lambda$ is not fully ascribed to the breakdown of adiabatic conditions since 
the Wigner delay times calculated by using Floquet scattering matrix are still much smaller than an oscillation period of external pumping potentials.

In Sec. II, we introduce our model system and investigate the characteristics of the pumped current using Brouwer's scattering approach. In Sec. III, we study the Floquet approach for parametric electron pumps and compare with those of Sec. II. We also discuss the validity of adiabatic theory by using the Wigner delay time. Finally, we conclude our paper in Sec. IV.

\section{BROUWER'S SCATTERING MATRIX APPROACH}

As a model system we consider $1 \mathrm{D}$ two harmonically oscillating $\delta$-function barriers with the strengthes $X_{1}=$ $V_{1}+\lambda_{1} \cos \omega \tau$ and $X_{2}=V_{2}+\lambda_{2} \cos (\omega \tau+\phi)$ respectively, separated by a distance $d$. This is a simplified model of the experiment by Switkes et al., but possesses many important characteristics and can be easily handled. Wei et al. studied parametric charge pumping aided by quantum resonance using this model and found that the pumped current has large values near a resonance level [19]. Due to the double barrier geometry, resonant tunneling also plays an important role in charge pumping.

The $2 \times 2$ scattering matrix of the double barriers with the strengthes $X_{1}$ and $X_{2}$ is given by

$$
S=\left(\begin{array}{cc}
r & t^{\prime} \\
t & r^{\prime}
\end{array}\right)
$$

where $r$ and $t$ are the reflection and the transmission amplitudes respectively, for modes incident from the left; $r^{\prime}$ and $t^{\prime}$ are similar quantities for modes incident from the right. The charge emitted per cycle to the right is obtained from

$$
Q_{1}=e \int_{0}^{T_{p}} d \tau\left(\frac{d n_{1}}{d X_{1}} \frac{d X_{1}}{d \tau}+\frac{d n_{1}}{d X_{2}} \frac{d X_{2}}{d \tau}\right)
$$

where

$$
\frac{d n_{1}}{d X_{m}}=\operatorname{Im}\left(\frac{\partial t}{\partial X_{m}} t^{*}+\frac{\partial r^{\prime}}{\partial X_{m}} r^{\prime *}\right)
$$

( $m=1,2)$, and $T_{p}(=2 \pi / \omega)$ is the period of the pumping. One can show that the emitted charge to the left $Q_{2}$ is equal to $-Q_{1}$. Equation (2) can be rewritten in the following form by using Green's theorem

$$
Q_{1}=e \int_{A} d X_{1} d X_{2} \Pi\left(X_{1}, X_{2}\right)
$$

where $\Pi\left(X_{1}, X_{2}\right)=\partial\left(d n_{1} / d X_{2}\right) / \partial X_{1}-\partial\left(d n_{1} / d X_{1}\right) / \partial X_{2}$. The pumped current is easily obtained from $I_{1}=Q_{1} / T_{p}$.

We use the parameters $d=50 \mathrm{~nm}$ for the distance between the two barriers, the effective mass $\mu=0.067 \mathrm{~m}_{e}$ of an electron in GaAs, and $T_{p}=9.09 \mathrm{ps}$ for the period of pumping, which corresponds to $\hbar \omega=0.45 \mathrm{meV}$. Figure 1 shows the pumped current as a function of the energy of an incident electron with $V_{1}=V_{2}=V, \lambda_{1}=\lambda_{2}=\lambda$, and $\phi=\pi / 2$. We present two examples; the first is a nearly open case $(V=0)$ in Figs. 1 $1 \mathrm{a}$ and $1 \mathrm{~b}$, and the second is a closed case $(V=225 \mathrm{meV} \cdot \mathrm{nm})$ in Figs. 11 $\mathrm{c}$ and 1 1 d. All of them show interesting resonance-like structures. In the closed case one can directly see the relation between the pumped currents and transmission resonances in Figs. 1 $\mathrm{i}$ and $1 \mathrm{j} \mathrm{d}$, where the transmission poles are denoted by the filled circles $(\bullet)$. To find these poles we considered a scattering problem with static double barriers of strength $V$. The imaginary part of the pole (denoted by the length of error bars attached to the filled circles) is related to the lifetime of a resonant (or quasibound) state, and determines the width of the transmission peaks. Peaks of the pumped current and their width are also described by the real and imaginary part of the resonance poles respectively, as shown in Fig. 1 $1 \mathrm{c}$. In Fig. 1 $1 \mathrm{~d}$, however, the width of the pumped current is larger than the imaginary part of the resonance energy due to the effect of strong oscillation. Moreover, in the open case (Figs. 1 $1 \mathrm{a}$ and $\mathrm{1} \mathrm{b}$ ), this analysis is no more relevant since there is no resonance in the static double barrier model.

Figure 2 shows the pumped current as a function of the pumping strength $\lambda$ for $V=0$ (the open case) with $\phi=\pi / 2$. Here for small $\lambda$ the pumped current depends on $\lambda^{2}$ and has an exact sinusoidal dependence on $\phi$, while for larger $\lambda$ the pumped current saturates and even decreases as $\lambda$ increases, and also deviates from the sinusoidal dependence on $\phi$. The decrease of the pumped current can be understood by considering the charge flux $\Pi\left(X_{1}, X_{2}\right)$ in Fig. 3. For small $\lambda$, the integration area only contains the positive $\Pi$ 's (the solid circle). Hence, as $\lambda$ increases $\int_{A} \Pi$ also becomes larger, which is roughly proportional to the area enclosed by the circle, i.e. $\int_{A} \Pi \propto \lambda^{2}$. Around $\lambda=225 \mathrm{meV} \cdot \mathrm{nm}$ 
(the dashed circle in Fig. 3) the integration area begins to include the region with both the positive and the negative $\Pi$ 's, which means $\int_{A} \Pi$ no longer increases as $\lambda$ increases. This explains why the pumped current saturates and even decreases above $\lambda=225 \mathrm{meV} \cdot \mathrm{nm}$ as shown in Fig. 2. From this we expect that the behavior of the saturation of the pumped current would depend on $\phi$ since the shape of the integration area is determined by $\phi$. For example, with $\phi=1.1 \pi$ the integration area denoted by the dashed dotted curve in Fig. 3 is distorted from the original circular shape, so that even for $\lambda=675 \mathrm{meV} \cdot \mathrm{nm}$ the integration area contains only positive $\Pi$ 's. Figure 2 clearly shows the pumped current with $\phi=1.1 \pi$ saturates much more slowly in comparison with the pumped current with $\phi=\pi / 2$. The non-sinusoidal dependence of the pumped current on $\phi$ for large $\lambda$ is also ascribed to the loss of circular symmetry of $\Pi$ in $\left(X_{1}, X_{2}\right)$.

\section{FLOQUET APPROACH}

Now we study the Floquet approach of the problem investigated in Sec. II. Using the scattering matrix of a single $\delta$-function with sinusoidal time dependence (see Appendix for details), we can obtain the total scattering matrix of the oscillating double $\delta$-functions in the following form

$$
S_{F l}=\left(\begin{array}{cc}
\mathbf{r} & \mathbf{t}^{\prime} \\
\mathbf{t} & \mathbf{r}^{\prime}
\end{array}\right)
$$

where $\mathbf{r}=\mathbf{r}_{L}+\mathbf{t}_{L}\left(\mathbf{I}-\mathbf{Q r}_{R} \mathbf{Q r _ { L }}\right)^{-1} \mathbf{Q r} \mathbf{r}_{R} \mathbf{Q t}$, and $\mathbf{t}=\mathbf{t}_{R}\left(\mathbf{I}-\mathbf{Q r}_{L} \mathbf{Q r _ { R }}\right)^{-1} \mathbf{Q} \mathbf{t}_{L} ; \mathbf{r}^{\prime}$ and $\mathbf{t}^{\prime}$ can also be obtained by replacing $L$ by $R$ in $\mathbf{r}$ and $\mathbf{t}$ respectively. Here $\mathbf{r}_{R(L)}$ and $\mathbf{t}_{R(L)}$ are the reflection and the transmission matrices respectively, for the right (left) delta function with time dependence for modes from the left, and $\mathbf{I}$ is an identity matrix. Due to the reflection symmetry of each delta function $\mathbf{r}_{R(L)}^{\prime}=\mathbf{r}_{R(L)}$, and $\mathbf{t}_{R(L)}^{\prime}=\mathbf{t}_{R(L)}$. During each one-way trip an electron at energy $E_{n}=E+n \hbar \omega$ picks up a phase factor $\exp \left(i k_{n} d\right)$, which is represented by the diagonal matrix $\mathbf{Q}_{m n}=\exp \left(i k_{m} d\right) \delta_{m n}$. From $S_{F l}$ we can obtain the total transmission coefficients for the propagating mode entering in the $m$ th channel

$$
T_{\rightarrow m}\left(E_{F l}\right)=\sum_{n=0}^{\infty}\left|\mathbf{t}_{n m}\right|^{2}
$$

where $E_{F l}$ is the Floquet energy. The total transmission coefficient from the left to the right as a function of an energy of an incident electron is given by $T_{\rightarrow}(E)=T_{\rightarrow m}\left(E_{F l}\right)$ where $E=E_{F l}+m \hbar \omega$. The total transmission from the right to the left $T_{\leftarrow}(E)$ can also be determined in a similar way.

The pumped current to the right is given by [17,20]

$$
I_{1}=\frac{2 e}{h} \int d E d E^{\prime}\left[t\left(E^{\prime}, E\right) f_{L}(E)-t^{\prime}\left(E, E^{\prime}\right) f_{R}\left(E^{\prime}\right)\right],
$$

where $t\left(E^{\prime}, E\right)$ represents the transmission probability for scattering states incident from the left at energy $E$ and emerging to the right at $E^{\prime}$, and $t^{\prime}\left(E, E^{\prime}\right)$ is defined in a similar manner for the reverse direction. $f_{L}\left(f_{R}\right)$ is the Fermi-Dirac distribution in the left (right) reservoir. Since the Floquet energy must be conserved during the scattering process, $E^{\prime}$ and $E$ are given by $E_{F l}+n \hbar \omega$ and $E_{F l}+m \hbar \omega$ respectively ( $n$ and $m$ are integers). Using Eq. (6), we can derive the relation $\int d E^{\prime} t\left(E^{\prime}, E\right)=\sum_{n} \mathbf{t}_{n m}\left(E_{F l}\right)=T_{\rightarrow}(E)$. Without external bias $\left(f_{L}=f_{R}=f\right)$ Eq. (7) can be rewritten as

$$
I_{1}=\frac{2 e}{h} \int_{0}^{\infty} d E f(E)\left[T_{\rightarrow}(E)-T_{\leftarrow}(E)\right]
$$

At zero temperature it becomes

$$
I_{1}=\frac{2 e}{h} \int_{0}^{E_{F}} d E\left[T_{\rightarrow}(E)-T_{\leftarrow}(E)\right],
$$

where $E_{F}$ is the Fermi energy. Equation (9) can also be expressed as $I_{1}=I_{\rightarrow}-I_{\leftarrow}$. This is quite interesting because from this point of view the pumped current merely corresponds to the difference of two currents having the opposite directions through a scatterer. 
Figure 1 shows that in the open case $(V=0)$ for small $\lambda$ (Fig. 1a) the pumped current obtained from the Floquet approach is equivalent to that of Brouwer's while for rather larger $\lambda$ (Fig. 五b) they deviate from each other. When $V \neq 0$ (the closed case), even for small $\lambda$ (Fig. 1 $1 \mathrm{c}$ ) they are quantitatively different near the resonances. Figure 1

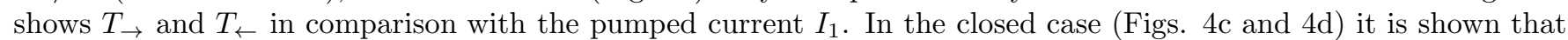
$T_{\rightarrow} \approx T_{\leftarrow}$, and the transmission resonances are directly related to the maxima of the pumped currents. In the open case (Figs. Aa and $4 \mathrm{~b}$ ), however, $T_{\rightarrow}$ and $T_{\leftarrow}$ considerably differ from each other, and it is hard to determine the relation between the resonances and the pumped currents. In fact, $T_{\rightarrow}$ looks out of phase with $T_{\leftarrow}$, and their resonance structures are quite complicated. In the open case the resonance-assisted electron pumping [19] seems unclear even though the oscillatory behavior of transmission looks similar to that of the pumped current.

As $\lambda$ increases with $V=0$, in Fig. 2, the pumped current obtained from the Floquet approach also saturates and even decreases although their exact values are different from those of Brouwer's. We also plot $I_{\rightarrow}$ and $I_{\leftarrow}$ in Fig. 2, where it is clear that in the Floquet approach the decrease of $I_{1}$ for large $\lambda$ is ascribed to the decrease of both $I_{\rightarrow}$ and $I_{\leftarrow}$. Usually the stronger the barrier strength, the smaller the transmission. This interpretation differs from that of Brouwer's, in which the decrease of the pumped current is explained by considering the structure of $\Pi\left(X_{1}, X_{2}\right)$ (Sec. II). Since we cannot define $\Pi\left(X_{1}, X_{2}\right)$ explicitly in the Floquet formalism, we plot $\Pi$ integrated over small circular area with a radius $\lambda$ centered at $\left(V_{1}, V_{2}\right)$. We consider only the case that $V_{1}=V_{2}=V$. Figure f shows that the integrated $\Pi$ 's obtained from the two different approaches for $\lambda=22.5 \mathrm{meV} \cdot \mathrm{nm}$ look very similar to each other, and their overall structure are governed by the static double barrier resonances denoted by the circles (o).

Brouwer's approach is based upon the adiabatic approximation, which implies that any time scale of the problem considered, especially the electron dwell time in a quantum dot (or inside double barriers in our case), must be much smaller than the period of the oscillation of a external pumping $T_{p}[\overline{[}]$. Using the Floquet formalism we can calculate the Wigner delay time $\tau_{W}$, which is the interaction time of the incident electron with the scattering potential [21] (see also [22,23]). In this sense $\tau_{W}$ corresponds to the electron dwell time in the quantum pump. To obtain the Wigner delay time we use the eigenvalues of the scattering matrix $S_{F l}$. Due to the unitarity of $S_{F l}$ all the eigenvalues lie on the unit circle and can be written in the form $\exp \left(i \theta_{\alpha}\right)$. The Wigner delay time is defined by

$$
\tau_{W}=\hbar \sum_{\alpha} \frac{d \theta_{\alpha}}{d E}\left|\left\langle k_{n} \mid \theta_{\alpha}\right\rangle\right|^{2}
$$

where the eigenstate corresponding to the eigenvalue $\theta_{\alpha}$ and an input propagating state (or channel) with momentum $k_{n}$ are denoted by $\left|\theta_{\alpha}\right\rangle$ and $\left|k_{n}\right\rangle$ respectively [24]. It is worth noting that the Wigner delay time is a function of the energy of the incident particle $E\left(=E_{F l}+n \hbar \omega\right)$, and $\left|k_{n}\right\rangle$ and $\left|\theta_{\alpha}\right\rangle$ are determined by $n$ and $E_{F l}$ respectively. If $\left\langle k_{n} \mid \theta_{\alpha}\right\rangle$ is ignored in Eq. 10 the Wigner delay time $\tau_{W}$ becomes trivial, i.e. $\tau_{W}(E+n \hbar \omega)=\tau_{W}(E)$. Thus, we cannot observe any signature of the double barrier resonances.

Figure 6 shows the Wigner delay time using the same parameters exploited in Fig. 0 . The Wigner delay times become smaller for both larger $E$ and lower $V$, which can be understood when we take into account that usually the electron dwell time is short if the energy of an incident electron is large or the scattering barrier is weak. The Wigner delay times have larger values near the resonances, which is ascribed to the fact that at the resonances an electron can stay in the quantum dot for a long time. Near the resonances the adiabatic condition can break down. This explains the deviation between the pumped currents observed in Figs. 다. In the open case $(V=0)$ we also check the Wigner delay time for rather larger $\lambda$ (up to $675 \mathrm{meV} \cdot \mathrm{nm}$ ), and observe they are smaller than $T_{p}$ by two orders of magnitude except for small incident energy $E$ (not shown), which means Brouwer's approach should be still applicable even for large $\lambda$. This leads us to conclude that the deviations of the pumped current observed in Figs. 1 1 a, $1 \mathrm{~b}$ and 2 for large $\lambda$ are not simply ascribed to the breakdown of adiabatic condition. It is worth noting that recently it is shown that Brouwer's formula is valid for arbitrary amplitudes of the modulating potential, as long as the lowest-order adiabatic approximation can be employed [11]. Let us just note that in Fig. 2 the discrepancy for large $\lambda$ appears when $\left|I_{1}\right| /\left|I_{\rightarrow}\right|$ (or $\left|I_{1}\right| /\left|I_{\leftarrow}\right|$ ) is not so small.

One of the interesting consequences from Eq. (9) is that the pumped current still exists even in the cases $\phi=0$ or $\pi$ when $\lambda_{1} \neq \lambda_{2}$ 18. Since the integration area in parameter space is zero when $\phi=0$ or $\pi$, in Brouwer's approach the pumped current definitely vanishes. In contrast, even when $\phi=0$ or $\pi$, an asymmetry of the potential can lead to the asymmetry of the currents [20], which is nothing but the pumped current in Eq. (9). Figure 7 shows the pumed current as a function of ratio of the strength of two barriers $\lambda_{2} / \lambda_{1}$ with $\phi=0$ or $\pi$, and $E=6.005 \hbar \omega$. Note that the pumped current is zero when $\lambda_{1}=\lambda_{2}$. The oscillatory behavior is also related to the double barrier resonances. 


\section{SUMMARY}

We investigate the Floquet scattering in parametric electron pumps in comparison with Brouwer's adiabatic scattering approach exploiting two harmonically oscillating $\delta$-function barriers. In the Floquet approach the pumped current simply corresponds to the difference of two currents having the opposite direction through a scatterer. For small strength of the oscillating potentials, these two kinds of approach give exactly equivalent results while for large strength these show deviation. Even though for large strength we obtain qualitatively similar results for the pumped currents using both approaches, the physical interpretation is completely different. The validity of the adiabatic approximation is also discussed by calculating Wigner delay time. For large $\lambda$ although the adiabatic condition is well satisfied, a quantitative discrepancy between both approaches is still observed. In the Floquet approach, a non-zero pumped current can be obtained even when $\phi=0$ or $\pi$ (no current at all in Brouwer's approach), if the spatial symmetry of the potential is broken $\left(\lambda_{1} \neq \lambda_{2}\right)$.

\section{ACKNOWLEDGMENTS}

We would like to thank Henning Schomerus and Hwa-Kyun Park for helpful discussion.

\section{APPENDIX:}

The scattering problem of a single $\delta$-function impurity with sinusoidal time dependence has been investigated by several authors 25 27. We would like to summarize how to construct its Floquet scattering matrix in this Appendix. The system is described by the Hamiltonian

$$
H(x, t)=-\frac{\hbar^{2}}{2 \mu} \frac{d^{2}}{d x^{2}}+\left[V_{s}+V_{d} \cos (\omega t+\phi)\right] \delta(x),
$$

where $\mu$ is the mass of the incident particle, while $V_{s}$ and $V_{d}$ represent the strength of the static and the oscillating potential respectively. Using the Floquet formalism the solution of this Hamiltonian can be expressed as

$$
\Psi_{E_{F l}}(x, t)=e^{-i E_{F l} t / \hbar} \sum_{n=-\infty}^{\infty} \psi_{n}(x) e^{-i n \omega t}
$$

where $E_{F l}$ is the Floquet energy which take continuous values in the interval $0<E_{F l} \leq \hbar \omega$.

Since the potential is zero everywhere except at $x=0, \psi_{n}(x)$ is given by the following form

$$
\psi_{n}(x)=\left\{\begin{array}{cc}
A_{n} e^{i k_{n} x}+B_{n} e^{-i k_{n} x}, & x<0 \\
C_{n} e^{i k_{n} x}+D_{n} e^{-i k_{n} x}, & x>0
\end{array}\right.
$$

where $k_{n}=\sqrt{2 \mu\left(E_{F l}+n \hbar \omega\right)} / \hbar$. The wave function $\Psi_{E_{F l}}(x, t)$ is continuous at $x=0$,

$$
A_{n}+B_{n}=C_{n}+D_{n}
$$

and the derivative jumps by

$$
\left.\frac{d \Psi_{E_{F l} l}}{d x}\right|_{x=0^{+}}-\left.\frac{d \Psi_{E_{F l}}}{d x}\right|_{x=0^{-}}=\frac{2 m}{\hbar^{2}}\left[V_{s}+V_{d} \cos (\omega t+\phi)\right] \Psi_{E_{F l}}(0, t) .
$$

Using Eq. (A2) this leads to the condition

$$
\begin{aligned}
& i k_{n}\left(C_{n}-D_{n}-A_{n}+B_{n}\right) \\
= & \gamma_{s}\left(A_{n}+B_{n}\right)+\gamma_{d}\left(e^{-i \phi} A_{n+1}+e^{i \phi} A_{n-1}+e^{-i \phi} B_{n+1}+e^{i \phi} B_{n-1}\right) \\
= & \gamma_{s}\left(C_{n}+D_{n}\right)+\gamma_{d}\left(e^{-i \phi} C_{n+1}+e^{i \phi} C_{n-1}+e^{-i \phi} D_{n+1}+e^{i \phi} D_{n-1}\right),
\end{aligned}
$$

where $\gamma_{s}=2 \mu V_{s} / \hbar^{2}$ and $\gamma_{d}=\mu V_{d} / \hbar^{2}$. After some algebra we have the following equation from Eqs. (A4) and (A6) 


$$
\left(\begin{array}{c}
\vec{B} \\
\vec{C}
\end{array}\right)=\left(\begin{array}{cc}
-(I+\Gamma)^{-1} \Gamma & (I+\Gamma)^{-1} \\
(I+\Gamma)^{-1} & -(I+\Gamma)^{-1} \Gamma
\end{array}\right)\left(\begin{array}{c}
\vec{A} \\
\vec{D}
\end{array}\right)
$$

where

$$
\Gamma=\left(\begin{array}{ccccc}
\ddots & \ddots & 0 & 0 & 0 \\
\gamma_{d} e^{i \phi} / i k_{-1} & \gamma_{s} / i k_{-1} & \gamma_{d} e^{-i \phi} / i k_{-1} & 0 & 0 \\
0 & \gamma_{d} e^{i \phi} / i k_{0} & \gamma_{s} / i k_{0} & \gamma_{d} e^{-i \phi} / i k_{0} & 0 \\
0 & 0 & \gamma_{d} e^{i \phi} / i k_{1} & \gamma_{s} / i k_{1} & \gamma_{d} e^{-i \phi} / i k_{1} \\
0 & 0 & 0 & \ddots & \ddots
\end{array}\right)
$$

and $I$ is an infinite-dimensional square identity matrix. Eq. (A7) can also be expressed in the form $\mid$ out $\rangle=M \mid$ in $\rangle$, where $M$ connects the input coefficients to the output coefficients including the associated evanescent Floquet sidebands. In order to construct the scattering matrix we multiply an identity to both sides, $K^{-1} K \mid$ out $\rangle=M K^{-1} K \mid$ in $\rangle$, where $K_{n m}=\sqrt{k_{n}} \delta_{n m}$. Then we have $\vec{J}_{\text {out }}=\bar{M} \vec{J}_{i n}$, where $\vec{J}$ represents the amplitude of probability flux and $\bar{M} \equiv K M K^{-1}$. It should be mentioned that $\bar{M}$ is not unitary due to the evanescent modes included.

If we keep only the propagating modes, we obtain the unitary scattering matrix $S$ [15, 16], which can be expressed in the following form

$$
S=\left(\begin{array}{cccccc}
r_{00} & r_{01} & \cdots & t_{00}^{\prime} & t_{01}^{\prime} & \cdots \\
r_{10} & r_{11} & \cdots & t_{10}^{\prime} & t_{11}^{\prime} & \cdots \\
\vdots & \vdots & \ddots & \vdots & \vdots & \ddots \\
t_{00} & t_{01} & \cdots & r_{00}^{\prime} & r_{01}^{\prime} & \cdots \\
t_{10} & t_{11} & \cdots & r_{10}^{\prime} & r_{11}^{\prime} & \cdots \\
\vdots & \vdots & \ddots & \vdots & \vdots & \ddots
\end{array}\right)
$$

where $r_{n m}$ and $t_{n m}$ are the reflection and the transmission amplitudes respectively, for modes incident from the left; $r_{n m}^{\prime}$ and $t_{n m}^{\prime}$ are similar quantities for modes incident from the right.

[1] D. J. Thouless, Phys. Rev. B 27, 6083 (1983).

[2] V. I. Talyanskii, J. M. Shilton, M. Pepper, C. G. Smith, C. J. B. Ford, E. H. Linfield, D. A. Ritchie, and G. A. C. Jones, Phys. Rev. B 56, 15180 (1997).

[3] L. P. Kouwenhoven, A. T. Johnson, N. C. van der Vaart, C. J. P. M. Harmans, and C. T. Foxon, Phys. Rev. Lett. 67, 1626 (1991).

[4] M. V. Berry, Proc. Roy. Soc. London, Ser. A 392, 45 (1984).

[5] B. L. Altshuler and L. I. Glazman, Science 283, 1864 (1999).

[6] J. E. Avron, A. Elgart, G. M. Graf, and L. Sadun, Phys. Rev. B 62, R10618 (2000).

[7] P. W. Brouwer, Phys. Rev. B 58, R10135 (1998).

[8] M. Switkes, C. M. Marcus, K. Campman, and A. C. Gossard, Science 283, 1905 (1999).

[9] G. B. Lubkin, Physics Today, June, p.19 (1999).

[10] F. Zhou, B. Spivak, and B. Altshuler, Phys. Rev. Lett. 82, 608 (1999).

[11] O. Entin-Wohlman, A. Aharony, and Y. Levinson, Phys. Rev. B 65, 195411 (2002).

[12] O. Entin-Wohlman and A. Aharony, Phys. Rev. B 66, 035329 (2002).

[13] M. Büttiker, H. Thomas, and A. Prêtre, Z. Phys. B 94, 133 (1994).

[14] H. Sambe, Phys. Rev. A 7, 2203 (1973).

[15] W. Li and L. E. Reichl, Phys. Rev. B 60, 15732 (1999).

[16] M. Henseler, T. Dittrich, and K. Richter, Phys. Rev. E 64, 046218 (2001).

[17] M. Wagner, Phys. Rev. Lett. 85, 174 (2000).

[18] S. -L. Zhu and Z. D. Wang, Phys. Rev. B 65, 155313 (2002).

[19] Y. Wei, J. Wang, and H. Guo, Phys. Rev. B 62, 9947 (2000).

[20] S. Datta and M. P. Anantram, Phys. Rev. B 45, 13761 (1992).

[21] E. P. Wigner, Phys. Rev. 98, 145 (1955). 
[22] F. Smith, Phys. Rev. 118, 349 (1960).

[23] Y. V. Fyodorov and H. -J. Sommers, J. Math. Phys. 38, 1918 (1997).

[24] A. Emmanouilidou and L. E. Reichl, Phys. Rev. A 65, 033405 (2002).

[25] P. F. Bagwell and R. K. Lake, Phys. Rev. B 46, 15329 (1992).

[26] D. F. Martinez and L. E. Reichl, Phys. Rev. B 64, 245315 (2001).

[27] S. W. Kim, H.-K. Park, H.-S. Sim, and H. Schomerus, cond-mat/0203391

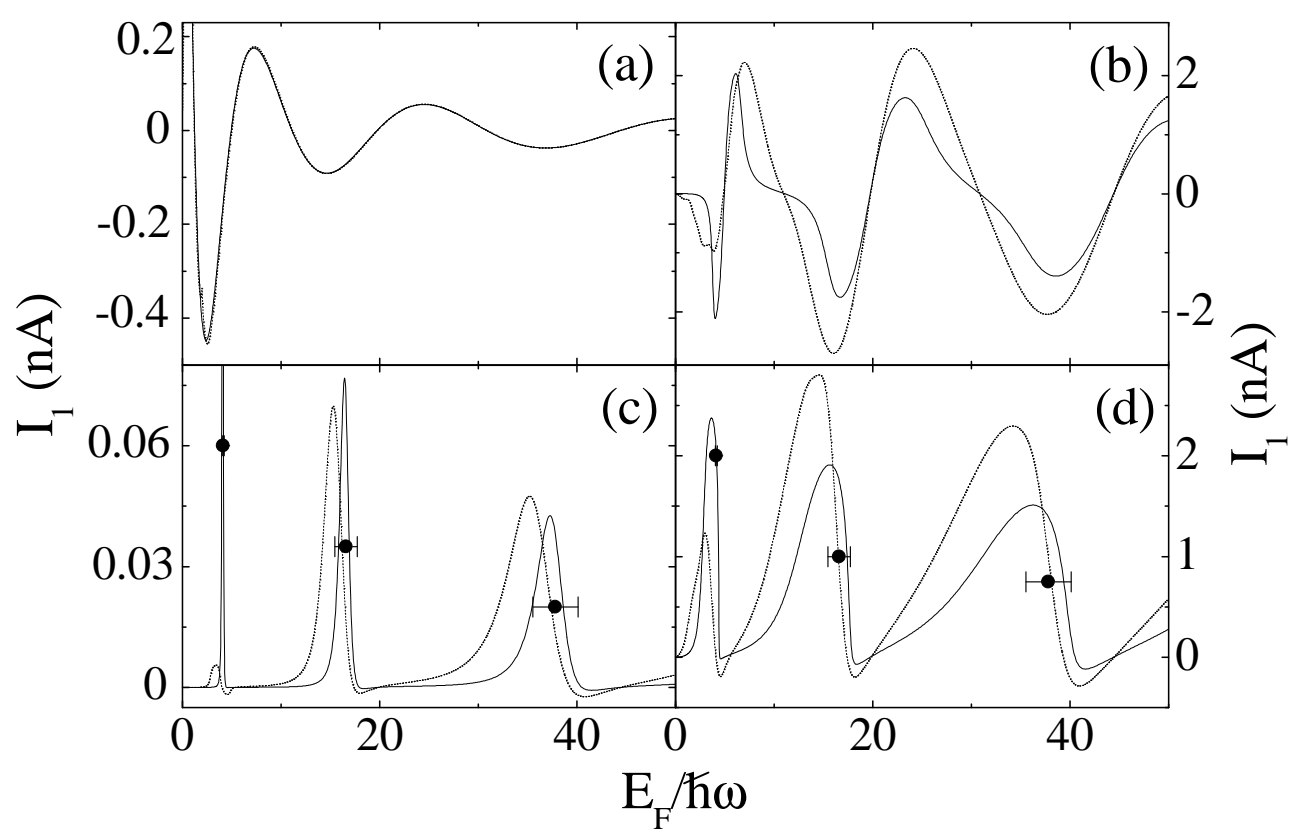

FIG. 1. The pumped current $I_{1}$ calculated by using Brouwer's approach (solid curves) and the Floquet approach (dotted curves) with $\phi=\pi / 2$ for (a) $\lambda=22.5 \mathrm{meV} \cdot \mathrm{nm}$ and (b) $\lambda=225 \mathrm{meV} \cdot \mathrm{nm}$ with $V=0$, and (c) $\lambda=22.5 \mathrm{meV} \cdot \mathrm{nm}$ and (d) $\lambda=225$ $\mathrm{meV} \cdot \mathrm{nm}$ with $V=225 \mathrm{meV} \cdot \mathrm{nm}$. In (c) and (d) the transmission resonances are denoted by the filled circles $(\bullet)$ obtained from considering static double barriers, whose $y$ values are chosen arbitrarily. The attached error bars represent the sizes of the imaginary energy of each resonance. 


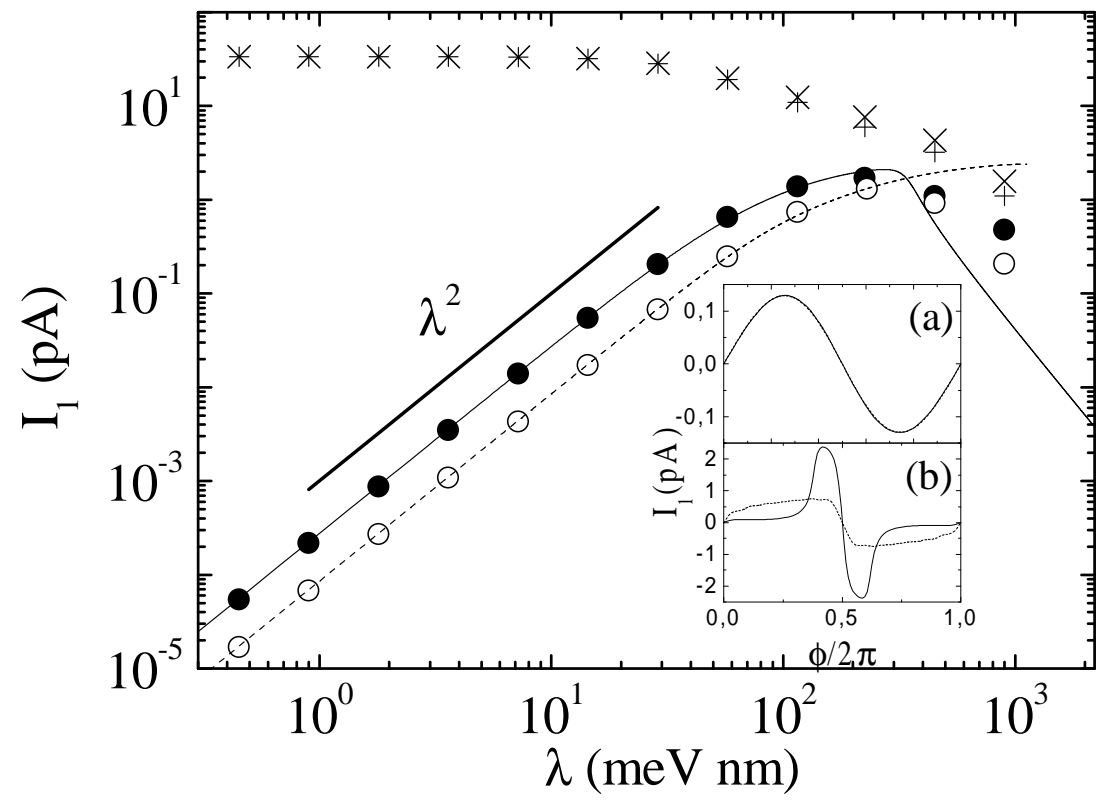

FIG. 2. The pumped current $I_{1}$ as a function of $\lambda$ with $V_{1}=V_{2}=0$ and $E=6.005 \hbar \omega$ by using Brouwer's approach for $\phi=\pi / 2$ (the solid curve) and $\phi=1.1 \pi$ (the dashed curve), and the Floquet approach for $\phi=\pi / 2(\bullet)$ and $\phi=1.1 \pi(\circ)$. $\times$ and + represent $I_{\rightarrow}$ and $I_{\leftarrow}$ for $\phi=\pi / 2$ respectively. The inset shows the pumped current $I_{1}$ as a function of $\phi$ for (a) $\lambda=22.5 \mathrm{meV} \cdot \mathrm{nm}$ and (b) $\lambda=675 \mathrm{meV} \cdot \mathrm{nm}$ by using Brouwer's approach (the solid curves) and the Floquet approach (the dotted curves).

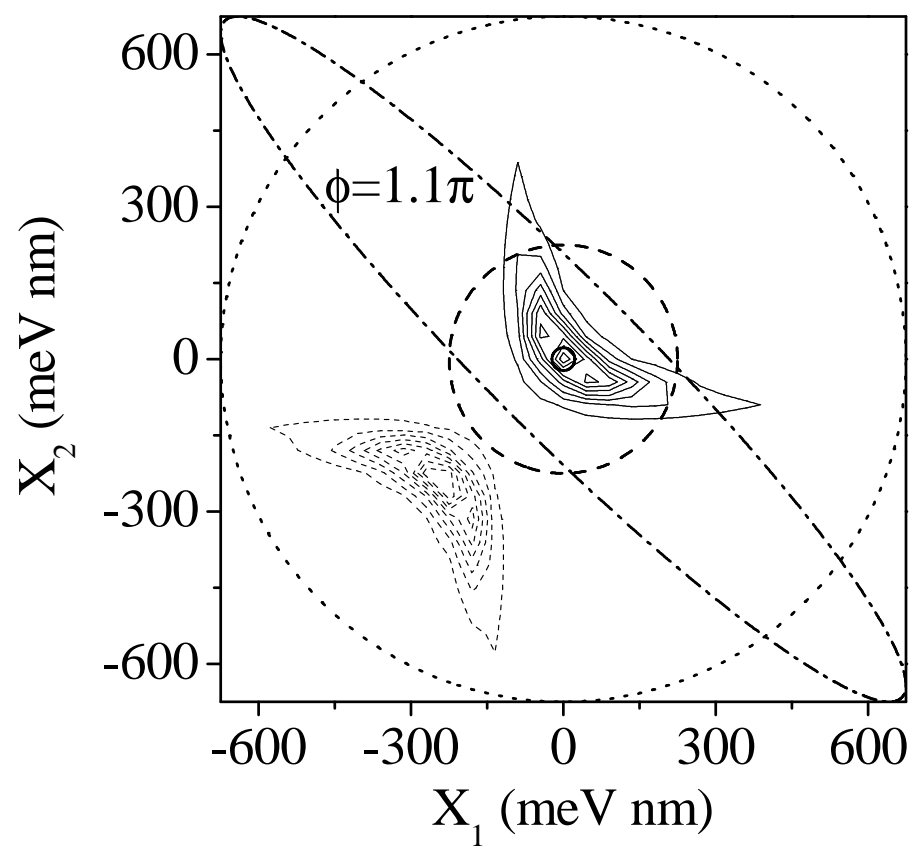

FIG. 3. Contour plot of flux $\Pi\left(X_{1}, X_{2}\right)$ for $E=6.005 \hbar \omega$. The solid and the dashed contours represent positive and negative fluxes respectively, in the range of -0.016 to 0.016 . The circles represent the integration area for $\phi=\pi / 2$ (see the text for detail) with $\lambda=22.5 \mathrm{meV} \cdot \mathrm{nm}$ (thick solid curve), $\lambda=225 \mathrm{meV} \cdot \mathrm{nm}$ (thick dashed curve), and $\lambda=675 \mathrm{meV} \cdot \mathrm{nm}$ (thick dotted curve). The dashed dotted curve represents the integration area with $\phi=1.1 \pi$ and $\lambda=675 \mathrm{meV} \cdot \mathrm{nm}$. 


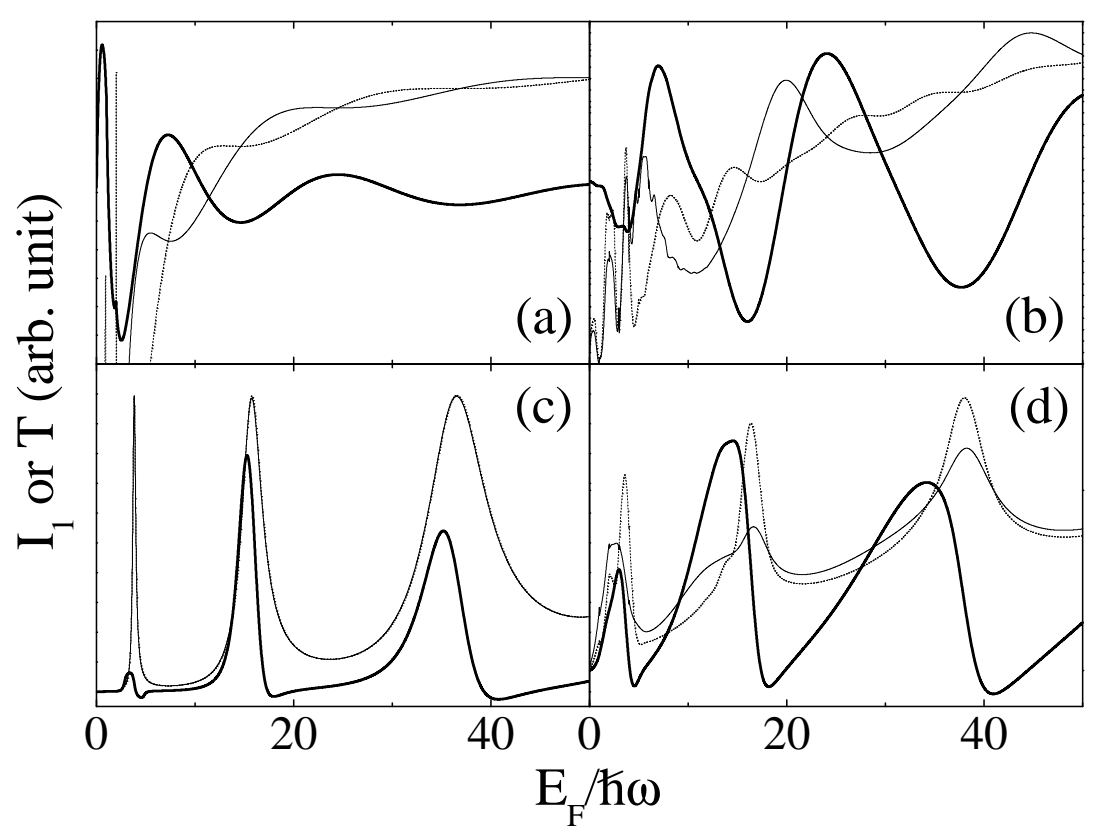

FIG. 4. Pumped current (thick solid curves), $T_{\rightarrow}$ (thin solid curves), and $T_{\leftarrow}$ (dotted curves) with the same parameters as used in Fig. I. For clear comparison the unit and the scale of the y-axis are arbitrarily chosen.

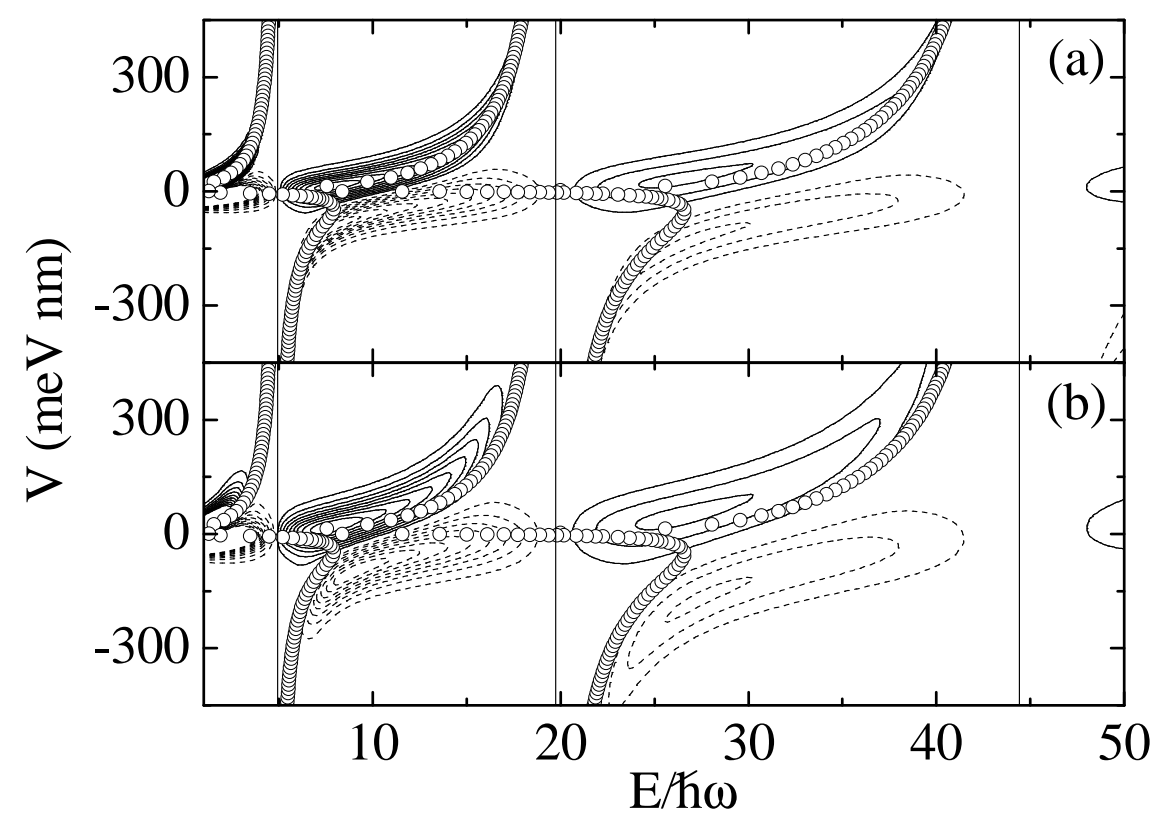

FIG. 5. Contour plot of the pumped current $I_{1}$ obtained from integrating $\Pi$ with $\lambda=22.5 \mathrm{meV} \cdot \mathrm{nm}$ (see the text for detail) by using (a) Brouwer's approach and (b) the Floquet approach. The solid and the dashed contours represent positive and negative currents respectively, in the range of $-0.2 \mathrm{nA}$ to $0.2 \mathrm{nA}$. The open circles (o) and the vertical lines represent the resonance energies of quasibound states of static double $\delta$-function barriers and the resonance energies from the condition $\pi^{2} \hbar^{2} m^{2} / 2 \mu d^{2}$ with an integer $m(m=1,2, \cdots)$ respectively. 

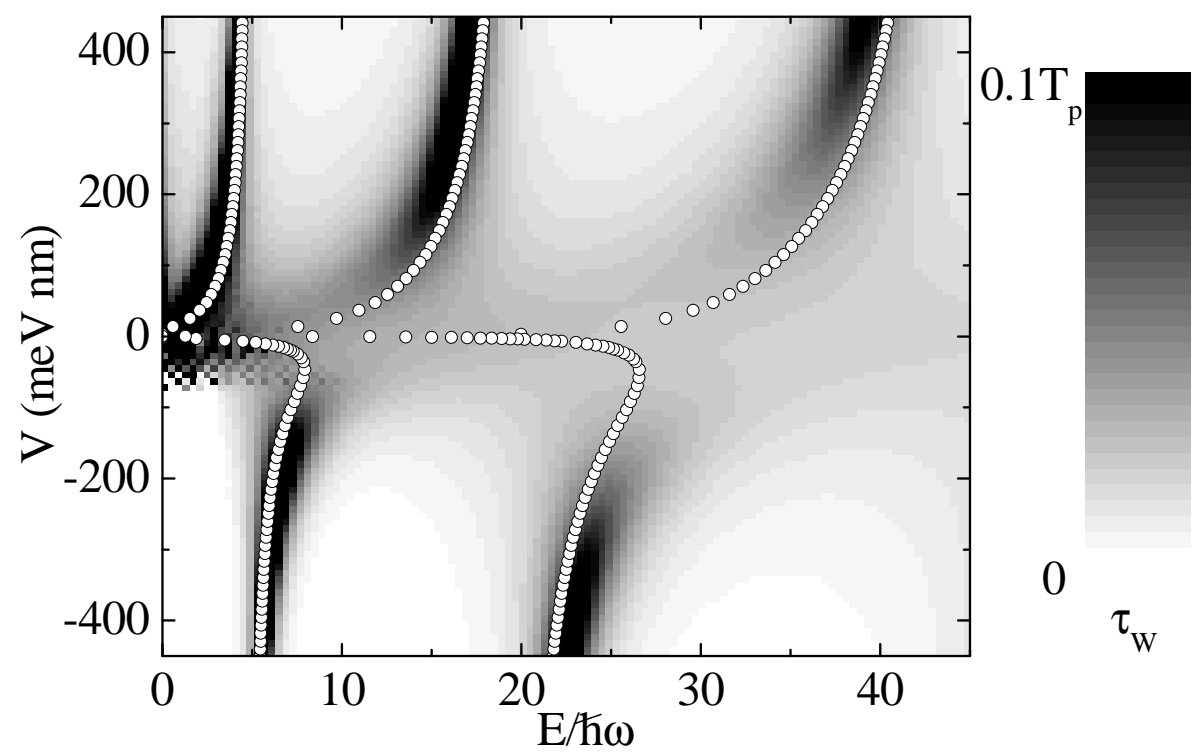

FIG. 6. Grey scale plot of the Wigner delay time. Black denotes times larger than $0.1 T_{p}$. The open circles (o) represent the resonance energies which are the same as in Fig. 5 .

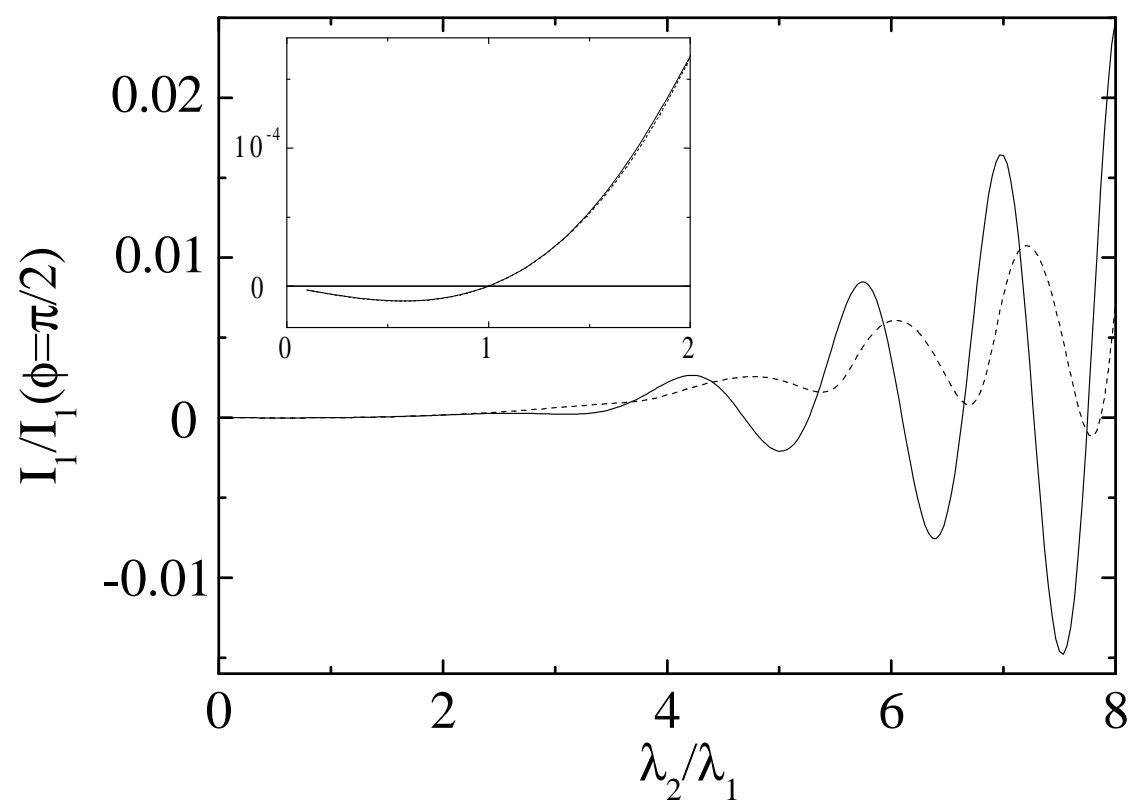

FIG. 7. Pumped current $I_{1}$ as a function of potential asymmetry $\lambda_{2} / \lambda_{1}$ with $\lambda_{1}=22.5 \mathrm{meV} \cdot \mathrm{nm}$ and $E=6.005 \hbar \omega$ at $\phi=0$ (solid curve) and $\phi=\pi$ (dashed curve), where the currents are normalized to their values at at $\phi=\pi / 2$. Inset shows the magnification of a part of the plot. 